\title{
The CG-BFGS method for unconstrained optimization problems
}

\begin{abstract}
In this paper we present a new search direction known as the CG-BFGS method, which uses the search direction of the conjugate gradient method approach in the quasi-Newton methods. The new algorithm is compared with the quasi-Newton methods in terms of the number of iterations and CPU-time. The Broyden-Fletcher-Goldfarb-Shanno (BFGS) method is used as an updating formula for the approximation of the Hessian for both methods. Our numerical analysis provides strong evidence that our CG-BFGS method is more efficient than the ordinary BFGS method. Besides, we also prove that the new algorithm is globally convergent.
\end{abstract}

Keyword: BFGS method; Conjugate gradient method; Search direction 